\title{
Editorial
}

\section{Green Composite Materials}

\author{
Hao Wang, ${ }^{1}$ Peter Schubel, ${ }^{2}$ Xiaosu Yi, ${ }^{3}$ Jin Zhu, ${ }^{4}$ Chad Ulven, ${ }^{5}$ and Yiping Qiu ${ }^{6}$ \\ ${ }^{1}$ Centre of Excellence in Engineered Fibre Composites, University of Southern Queensland, Toowoomba, QLD 4350, Australia \\ ${ }^{2}$ Division of Materials, Mechanics and Structures, University of Nottingham, Nottingham NG96PA, UK \\ ${ }^{3}$ Beijing Institute of Aeronautical Materials, Beijing 100095, China \\ ${ }^{4}$ Ningbo Institute of Industrial Technology, Chinese Academy of Sciences, Ningbo 315201, China \\ ${ }^{5}$ North Dakota State University, Fargo, ND 58108-6050, USA \\ ${ }^{6}$ College of Textiles, Donghua University, Shanghai 201620, China
}

Correspondence should be addressed to Hao Wang; hao.wang@usq.edu.au

Received 16 May 2015; Accepted 18 May 2015

Copyright (C) 2015 Hao Wang et al. This is an open access article distributed under the Creative Commons Attribution License, which permits unrestricted use, distribution, and reproduction in any medium, provided the original work is properly cited.

Global awareness of environmental issues has resulted in the emergence of sustainable and environmentally friendly green materials, which are renewable resources based, recyclable, and biodegradable. These green composite materials can be polymer based or cement based. In the polymer based green composites, natural fibres, such as hemp, flax, jute, kenaf, and sisal, have been used to replace conventional synthetic fibres. On the other hand, bioresins have been derived from starch, vegetable oils, and protein to replace petroleum based polymers. Cement based composites utilise new binders, such as geopolymer and recycled aggregators. The use of renewable and recycled resources reduces the need for petrochemicals and minerals, resulting in reduced natural resources depletion. Commercial products and applications are now emerging for these green composites.

Green composites are regarded as the next generation of sustainable composite materials, gaining significant attention from both academia and industry. This special issue is just a snapshot to present some of the latest progress in green composites fabrication, characterisation, testing, and applications.

The special issue contains eight papers on polymer based green composites, including hemp, flax, and sisal fibre reinforced composites, and polylactic acid and chitosan biopolymers. It also contains five papers on cement based composites, including geopolymers from slag, fly ash, metakaolin, and rice hush ash. There are also papers across these two areas, which use oil palm shell and waste cellulose in cement and concrete.
We hope the information provided in this special issue is useful and offers stimulation to the new development of green composite materials and the promotion of these materials to industrial applications.
Hao Wang Peter Schubel

Xiaosu Yi Jin Zhu

Chad Ulven Yiping Qiu 

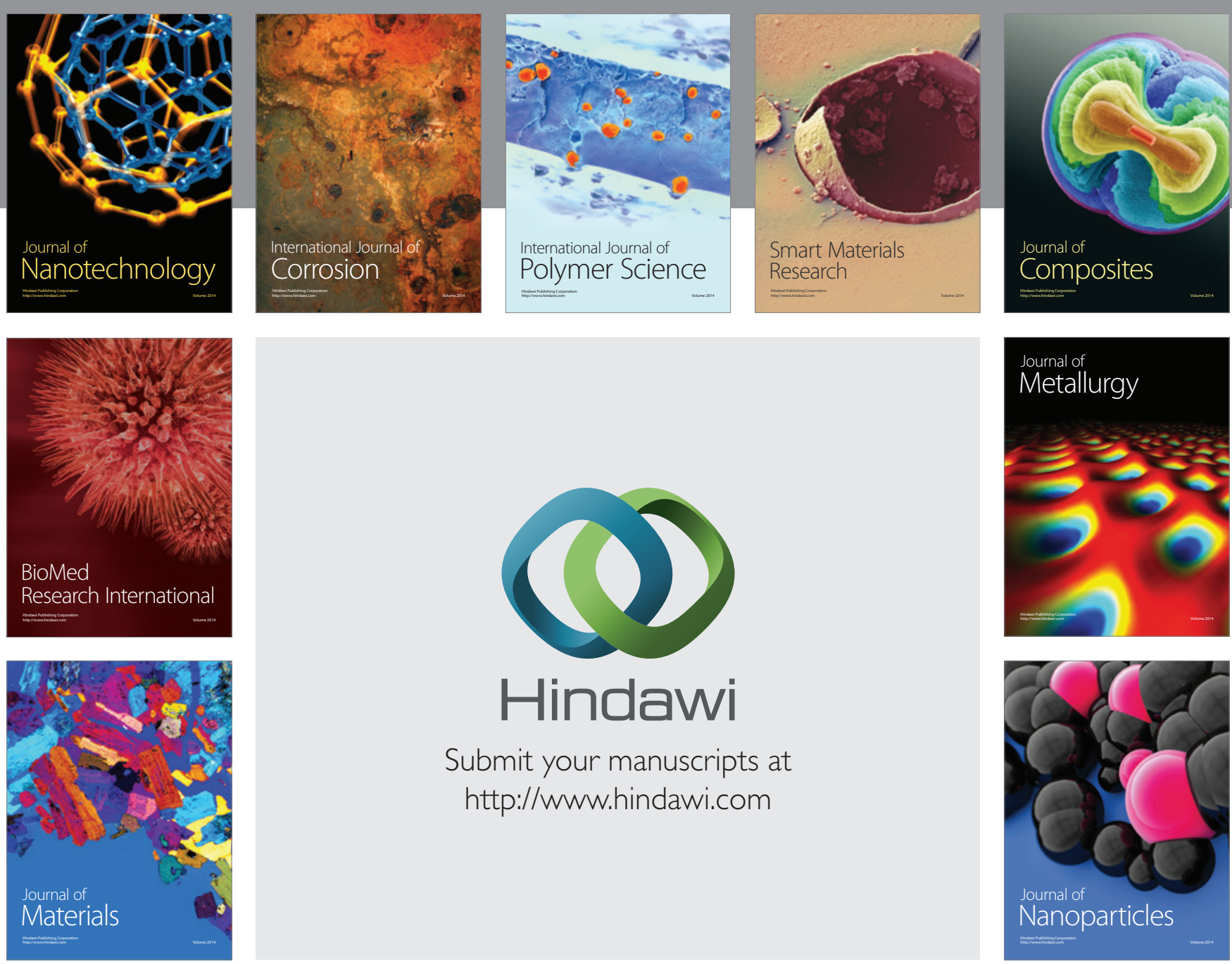

Submit your manuscripts at http://www.hindawi.com
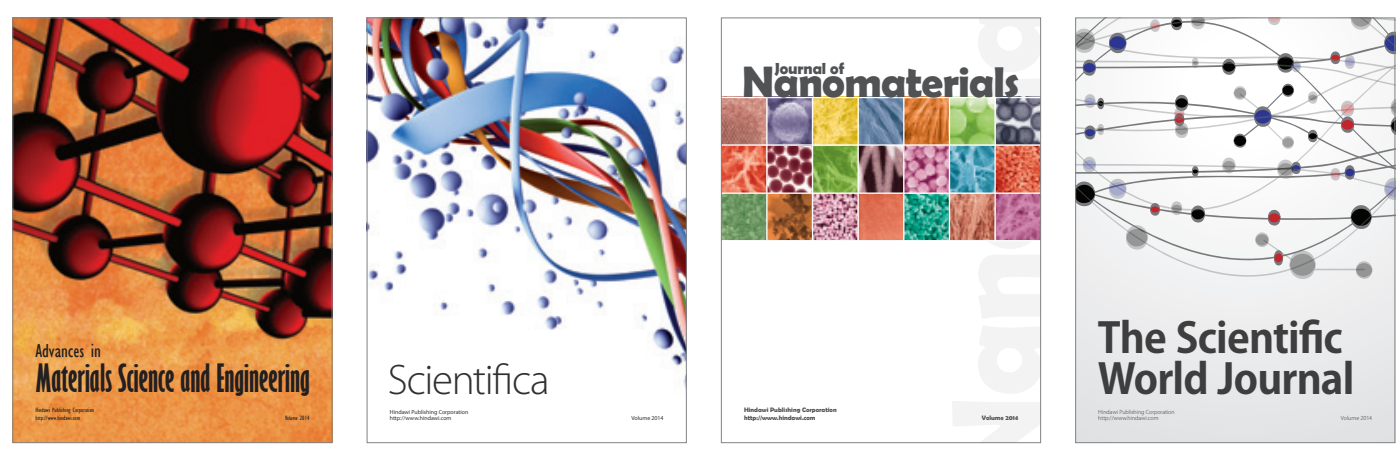

\section{The Scientific World Journal}
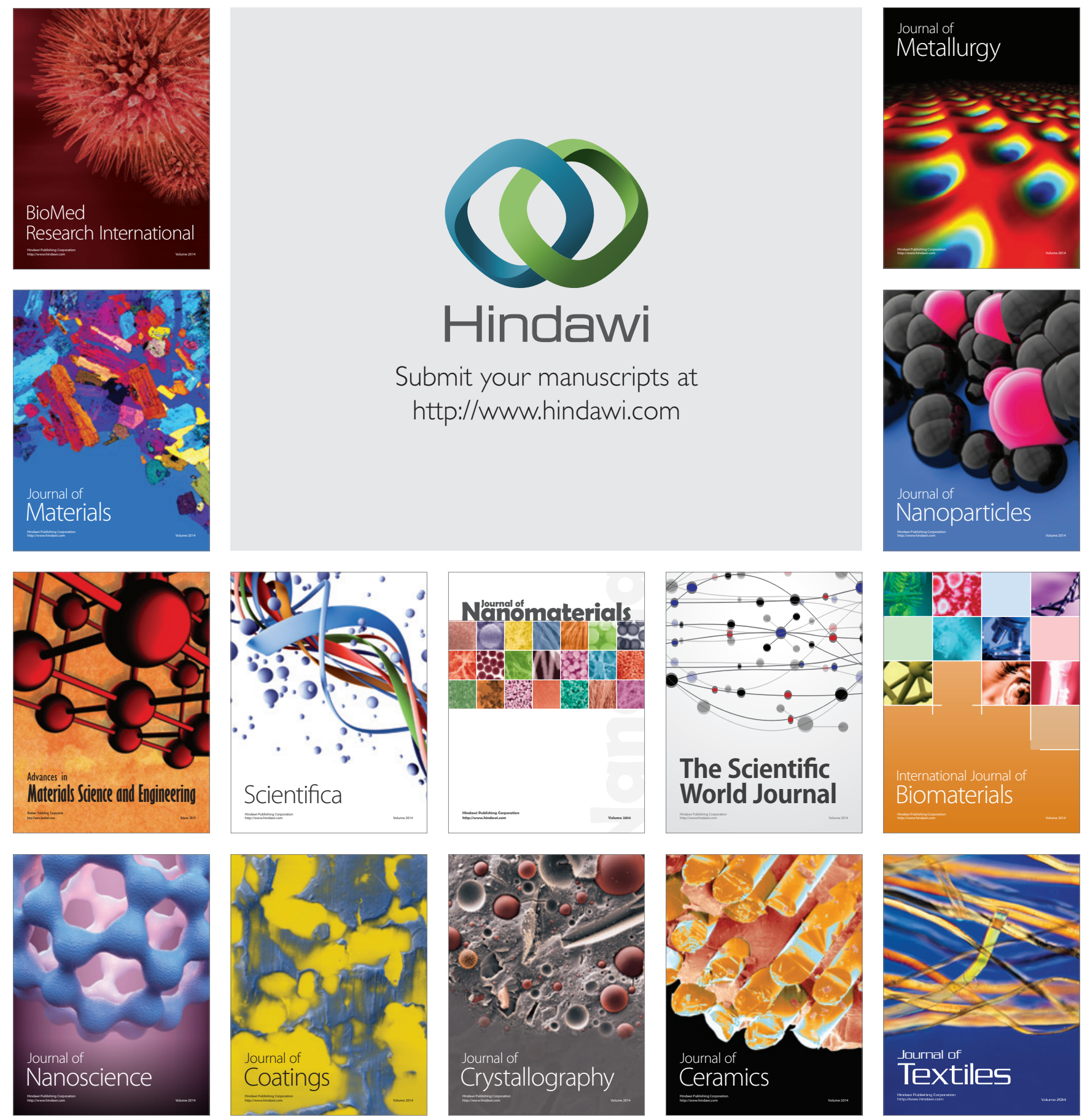